\title{
Wind Turbine Seismic Load Analysis Based on Numerical Calculation
}

\author{
Xin Jin ${ }^{1, *}$ - Hua Liu ${ }^{2}-$ Wenbin Ju1 \\ ${ }^{1}$ Chongqing University, College of Mechanical Engineering, China \\ 2 Dongfang Electric Corporation, China
}

\begin{abstract}
Large-scale wind turbines have come into common use in Europe. Because violent earthquakes are relatively rare there, insufficient consideration has been given to the seismic impact on the wind turbine specifications; however, at present, there are many wind farms being constructed in earthquake-prone regions, and the seismic impact cannot be ignored in wind turbine designs. Based on the multi-body system dynamic theory and taking into consideration the soil-structure interaction, this paper proposes a blade-cabin-tower-foundation coupled model in order to study the load-bearing conditions of wind turbines under seismic impact. According to the basic theory of multi-body system dynamics, the wind turbine blade and tower system comprises a series of continuous discrete units, while soil-structure interaction in the tower system is realized through the spring and damping set on the interface between the foundation and the soil body; the cabin is simplified as a rigid model. Based on the Eurocode 8 earthquake load spectrum, the dynamic response of a wind turbine working under seismic impact is analysed, and the seismic load is compared. Results of the study can serve as references for designing key parts and control strategies of wind turbines for earthquake-prone regions.
\end{abstract}

Keywords: wind turbine, earthquake, multi-body dynamics, soil-structure interaction

\section{INTRODUCTION AND BACKGROUND}

As the price of wind power drops, wind turbines are playing an increasingly important role in the global power supply. In recent decades, wind turbine systems have been mainly established in North Europe, which is not earthquake-prone (or merely subject to less violent earthquakes), and thus seismic impact has not been focussed on in the specifications for wind turbine systems [1]. However, increasing numbers of wind turbine systems are being constructed in earthquakeprone regions; therefore, it is necessary to analyse the dynamic response characteristics of wind turbine systems to earthquakes, and to consider the impact of seismic load in further engineering designs.

There are some relevant studies regarding wind turbines. For example, Teng et al. [2] used empirical mode decomposition on the pitting fault detection of a wind turbine gearbox. Potočar et al. [3] used plasma actuators to control separation flow over a wind turbine blade. However, there are limited studies on wind turbines under seismic impact. The Risø National Laboratory of Denmark [4] analysed the seismic load on wind turbines by using the first-order natural vibration frequency. In 2002, Bazeos et al. [5] improved the model established by his predecessors and created a wind turbine tower model. The tower body was modelled to be of three sections, with the size gradually increasing from the top to the bottom; each section had the same size, with a progressive transition between adjoining sections. Bazeos et al. used the time-history method to analyse the dynamic seismic load, and then considered the impact of soilstructure interaction, and finally concluded that the soil-structure interaction had an obvious impact on the overall system. In 2003, Lavassas et al. [6] proposed another wind turbine finite element model, in which the tower body was a truncated cone shell with a pile base at the bottom; in this model, the authors simulated the impact of soil-structure interaction by setting a contact element between the foundation and the soil body. The common point of the two models above was to model the tower body and the foundation, but not the blade and cabin; therefore, it was difficult to use them for the refined analysis and design of the wind turbine tower system. Murtagh et al. [7] and [8] proposed a shear transfer-based blade and tower coupling finite element model, having clarified the coupling mechanism of the blade and tower body and used the time-history method to analyse the dynamic wind load on the structure. Nevertheless, the authors did not model the foundation, nor considered the effect of soil-structure interaction. Witcher [9] studied the seismic load and applied the seismic analysis method for the bridge and building structures to wind turbines, but he did not consider the soil-structure interaction. Kang et al. [10] used a nonlinear spring to simulate the soil-structure interaction and analysed the reliability of offshore wind turbine bases, but the applied load was static. Harte et al. [11] studied the wind turbine response to wind-induced buffeting and considered the soil-structure interaction in the model, but he did not carry out a seismic analysis. Lombardi et al. [12] conducted a series of experiments on the 
soil-structure interaction of wind turbines, in which only the natural vibration frequency of the structure was considered; however, no consideration was given to the wind load borne by the wind turbines, nor was any seismic analysis carried out. It is easy to conclude that to date there are few studies on modelling bladetower-foundation coupled with a multi-body system of wind turbines, particularly by using the time-history method to analyse the dynamic seismic load based on the coupled model.

In consideration of the above, this paper uses the multi-body system dynamic theory as a basis for studying the dynamic response of wind turbines to earthquakes, while taking into account the soilstructure interaction. According to the basic theory of multi-body system dynamics, the wind turbine blade and tower system comprises a series of continuous, discrete units, while the soil-structure interaction of the wind turbine tower system is realized through the spring and damping set on the interface between the foundation and the soil body. In order to study the dynamic characteristics of a wind turbine, the time-history method is used to analyse the dynamic seismic impact on the wind turbine based on Eurocode 8 , and an analysis model is established to study the seismic impact on load-bearing conditions of the wind turbine during operation, so as to provide references for designing key parts and control strategies of wind turbines for earthquake-prone regions.

\section{COORDINATE SYSTEMS}

Coordinate systems are critical in structural analysis. A proper coordinate system may function to simplify calculations; therefore, it is necessary to establish a wind turbine coordinate system before establishing a structural analysis model. As required for analysis, a dynamic coordinate system for a three-blade wind turbine can be established, as shown in Fig. 1. Fig 1a shows the inertia system $Z$ (orthogonal coordinate axes $z_{1}, z_{2}$ and $z_{3}$ ), wind turbine base system $A$ (orthogonal coordinate axes $a_{1}, a_{2}$ and $a_{3}$ ), tower system $T$ (orthogonal coordinate axes $t_{1}, t_{2}$ and $t_{3}$ ), tower top system $B$ (orthogonal coordinate axes $b_{1}$, $b_{2}$ and $b_{3}$ ). Fig $1 \mathrm{~b}$ shows the principal axes system $C$ (orthogonal coordinate axes $c_{1}, c_{2}$ and $c_{3}$ ), rotor azimuth system $E$ (orthogonal coordinate axes $e_{1}, e_{2}$ and $e_{3}$ ), hub system $G$ (orthogonal coordinate axes $g_{1}$, $g_{2}$ and $g_{3}$ ), cone angle system $I$ (orthogonal coordinate axes $i_{1}, i_{2}$ and $i_{3}$ ), blade pitch system $J$ (orthogonal coordinate axes $j_{1}, j_{2}$ and $j_{3}$ ), blade local torsion angle system $L j$ (orthogonal coordinate axes $L j_{1}, L j_{2}$ and $L j_{3}$ ), and blade element local torsion angle system $N$ (orthogonal coordinate axes $n_{1}, n_{2}$ and $n_{3}$ ) etc. The details of the coordinate system $J, L j$ and $N$ are shown in Fig. 1c.

\section{DISCRETENESS MODEL}

Suppose the wind turbine blade and tower system comprises a flexible cantilever with evenly and continuously distributed mass and rigidity, as shown in Fig. 2. Deformation of the continuous blade and tower system is expressed with a series of linearly overlapped normalized vibration modes; so that the freedom of the blade and tower is reduced from infinity to $N$ ( $N$ is the supposed number of modes selected for calculation). Then the horizontal deformation $u(z, t)$ of

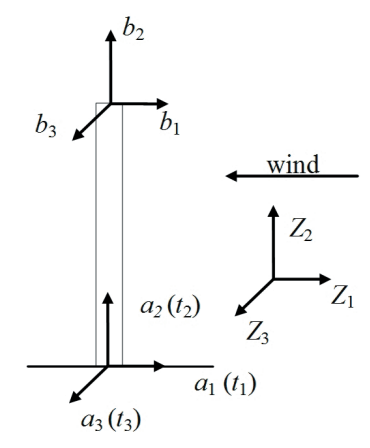

a)

b)

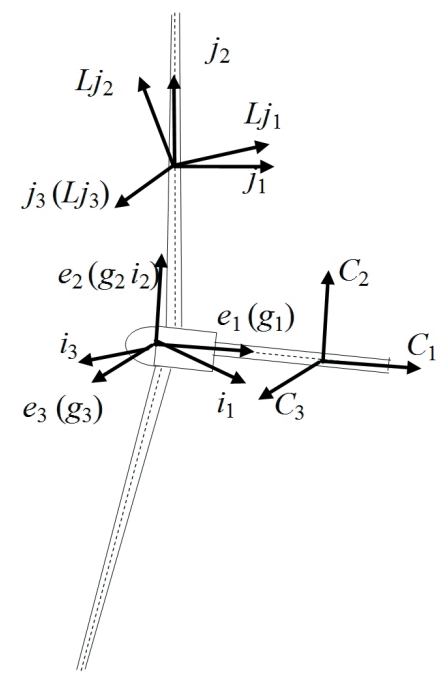

c)

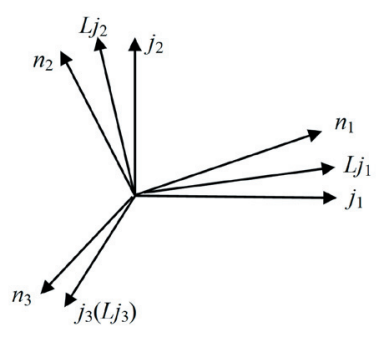

Fig. 1. Coordinate systems; a) tower coordinate systems b) drivetrain coordinate systems c) detail coordinate system of blade 
the flexible cantilever, at any time and any place, is a series of linearly overlapped normalized modes $\phi_{a}(z)$, which associate with the generalized coordinates $q_{a}(t)$ as follows:

$$
u(z, t)=\sum_{a=1}^{N} \phi_{a}(z) q_{a}(t),
$$

where $\phi_{a}(z)$ is the ath mode of the cantilever and a function of the cantilever longitudinal distance $z$; the generalized coordinate $q_{a}(t)$ associating with the mode $a$ is a function of time, and it is commonly the free end deformation of the cantilever corresponding to the mode.

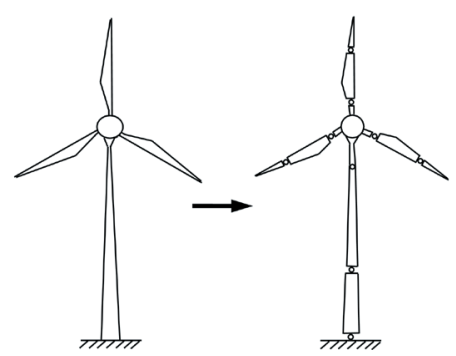

Fig. 2. Discreteness model

By using the Lagrange equation of a conservative and constant system, the kinetic equation of $N$ freedom systems can be expressed as:

$$
\begin{gathered}
\sum_{j=p}^{N+p-1} m_{i j} \ddot{c}_{j}(t)+\sum_{j=p}^{N+p-1} k_{i j} \dot{c}_{j}(t)=0, \\
(i=p, p+1, \cdots, N+p-1),
\end{gathered}
$$

where the generalized mass $m_{i j}$ and the generalized rigidity $k_{i j}$ can be defined by using the kinetic energy $T$ and potential $V$ as:

$$
\begin{gathered}
T=\frac{1}{2} \sum_{i=p}^{N+p-1} \sum_{j=p}^{N+p-1} m_{i j} \dot{c}_{i}(t) \dot{c}_{j}(t), \\
V=\frac{1}{2} \sum_{i=p}^{N+p-1} \sum_{j=p}^{N+p-1} k_{i j} c_{i}(t) c_{j}(t) .
\end{gathered}
$$

When the cantilever vibrates at a specific inherent frequency $\omega_{a}$, suppose $a=m$, we can obtain:

$$
q_{a}(t)=\left\{\begin{array}{cc}
Q_{a} \sin \left(\omega_{a} t+\psi_{a}\right) & a=m \\
0 & a \neq m
\end{array}\right.
$$

where $Q_{a}$ is the deformation of the cantilever free-end in the free mode $a$. By applying $q_{a}(t)$ in the equation $C_{b}(t)=C_{m, b} q_{m}(t)$, and applying the result obtained from the foregoing equation in Eq. (5), we can obtain:

$$
\begin{gathered}
\sum_{j=p}^{N+p-1}\left(-\omega^{2} m_{i j}+k_{i j}\right) C_{j}=0, \\
(i=p, p+1, \cdots, N+p-1),
\end{gathered}
$$

which can be transformed to be a matrix as follows:

$$
\left(-\omega^{2}[\mathbf{M}]+[\mathbf{K}]\right)\{\mathbf{C}\}=\{\mathbf{0}\},
$$

where the generalized mass matrix $[\mathbf{M}]$ and the generalized rigidity matrix $[\mathbf{K}]$ are $N \times N$ order matrixes; the coefficient vector $\{\mathbf{C}\}$ is an $N \times 1$ order vector. By analysing the characteristic values of the matrix equation, we can obtain the characteristic value $\omega^{2}{ }_{a}$ and the characteristic vector $\{\mathbf{C}\}_{a}$.

\subsection{Tower Modelling}

The tower is simulated to be an inverted cantilever whose free end is fixed with a point mass $M_{\text {Top }}$, representing the total mass of the base plate, the cabin, the hub and the blade. The generalized mass of the tower can be expressed as:

$$
m_{i j}=M_{T o p}+\int_{0}^{H} \mu_{T}(h) \phi_{i}(h) \phi_{j}(h) d h,
$$

where $\mu_{T}(h)$ is the mass distribution line density of the tower.

The tower potential comprises $V_{\text {Beam }}$ associating with distribution rigidity of the beam and $V_{\text {Gravity }}$ associating with gravity.

$$
V=V_{\text {Beam }}+V_{\text {Gravity }},
$$

where the potential component associating with the distribution rigidity of the cantilever can be expressed as:

$$
V_{\text {Beam }}=\frac{1}{2} \sum_{i=p}^{N+p-1 \sum_{j=p}^{N+p-1}}\left(\int_{0}^{H} E I_{T}(h) \frac{d^{2} \phi_{i}(h)}{d h^{2}} \frac{d^{2} \phi_{j}(h)}{d h^{2}} d h\right) c_{i}(t) c_{j}(t),
$$

where $E I_{T}(h)$ is the distribution rigidity of the tower and $H$ is the height of the tower.

The potential component associating with gravity can be expressed as:

$$
V_{\text {Gravity }}=-\frac{1}{2} g \sum_{i=p}^{N+p-1} \sum_{j=p}^{N+p-1}\left(M_{\text {Top }} \int_{0}^{H} \frac{d \phi_{i}(h)}{d h} \frac{d \phi_{j}(h)}{d h} d h+\int_{0}^{H} \mu_{T}(h)\left(\int_{0}^{h} \frac{d \phi_{i}\left(h^{\prime}\right)}{d h^{\prime}} \frac{d \phi_{j}\left(h^{\prime}\right)}{d h^{\prime}} d h^{\prime}\right) d h\right) c_{i}(t) c_{j}(t),
$$


where the minus sign indicates that gravity will reduce the generalized rigidity of the tower; the first item in the bracket is associated with gravity of the tower mass, and the second item is associated with gravity of the tower distribution mass, with the impact of the tower deformation on the gravitational potential considered.

The generalized rigidity of the tower can be expressed as:

$$
\begin{aligned}
k_{i j} & =\int_{0}^{H} E I_{T}(h) \frac{d^{2} \phi_{i}(h)}{d h^{2}} \frac{d \phi_{j}(h)}{d h^{2}} d h- \\
& -g \int_{0}^{H}\left[M_{T o p}+\int_{h}^{H} \mu_{T}\left(h^{\prime}\right) d h^{\prime}\right] \frac{d \phi_{i}(h)}{d h} \frac{d \phi_{j}(h)}{d h} d h .
\end{aligned}
$$

\subsection{Soil-Structure Interaction Modelling}

The interaction between the soil and the foundation is an interactive force on the contact surface, which is caused by different material properties of the soil body and the structure (mainly the elastic modulus). In civil engineering, that the soil-structure interaction impacts the dynamic response of structures is a generally known phenomenon. For wind turbine towers established on soft soil (Fig. 3), the soilstructure interaction is considered to be one of the key factors in dynamic analysis, and a more critical factor for wind turbine tower systems in earthquake-prone regions.

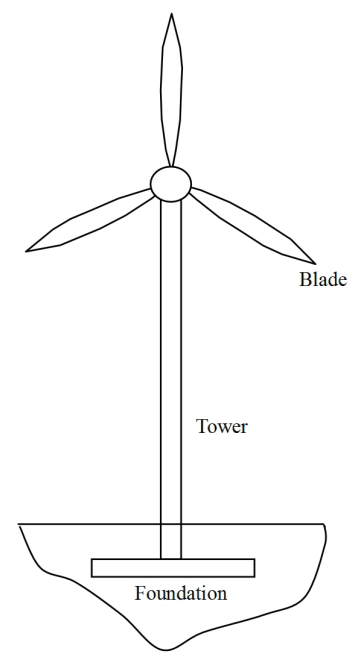

Fig. 3. Wind turbine system

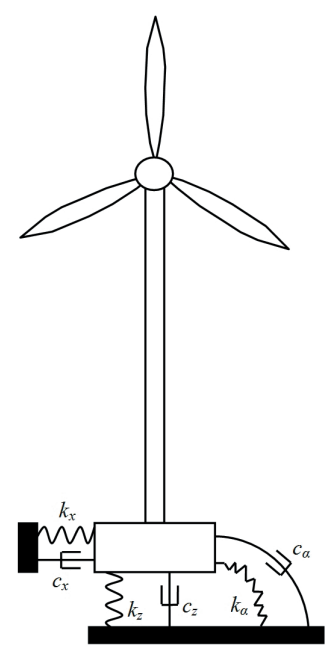

Fig. 4. Dynamic model of soil-structure interaction
The continuum medium model (analytical method), the discrete model and the finite element model can be used to study complex soil-structure interactions. However, the continuum medium model is too complex, while the finite element model used to simulate soil-structure interactions is overly time-consuming and is thus disadvantageous for calculation. Therefore, to comprehend the most essential characteristics of soil-structure interaction in wind turbine tower systems, one simple and effective approach is to set a spring and damping on the interface between the foundation and the soil body (Fig. 4).

For a three-dimensional soil-structure interaction model, the two horizontal displacements couple with the rotary movement. However, the coupling item can be neglected as it is of a relatively small value [13]; this is particularly the case for wind turbine tower systems based in shallow soil (Fig. 2). Therefore, all dynamic components are considered to not be subject to mutual coupling. For a rigid round foundation, the rigidity and damping coefficient can be determined based on the properties of the surrounding soil body and the foundation size [14] to [16], which can be expressed as:

$$
\begin{gathered}
\left\{\begin{array}{c}
k_{x}=k_{y}=\frac{8 G_{s} R_{s}}{2-\mu_{s}} \\
k_{z}=\frac{4 G_{s} R_{s}}{1-\mu_{s}} \\
k_{\alpha}=k_{\beta}=\frac{8 G_{s} R_{s}^{3}}{3\left(1-\mu_{s}\right)} \\
k_{\gamma}=\frac{16}{3} G_{s} R_{s}^{3} \\
c_{x}=c_{y}=\frac{4.6 R_{s}^{2}}{2-\mu} \sqrt{G_{s} \rho_{s}} \\
c_{z}=\frac{3.4 R_{s}^{2}}{1-\mu} \sqrt{G_{s} \rho_{s}} \\
c_{\alpha}=c_{\beta}=\frac{0.4 R_{s}^{4}}{1-\mu} \sqrt{G_{s} \rho_{s}} \\
c_{\gamma}=1.11 R_{s}^{4} \sqrt{G_{s} \rho_{s}}
\end{array}\right.
\end{gathered}
$$

where $k_{x}$ and $k_{y}$ are horizontal rigidity coefficients; $k_{z}$ is the vertical rigidity coefficient; $k_{\alpha}$ and $k_{\beta}$ are the bending rigidity coefficients; $k_{\gamma}$ is the bending coefficient about the vertical axis. Similarly, $c_{i}(i=x$, $y, z, \alpha, \beta, \gamma)$ is the corresponding damping coefficient. $R_{s}$ is the round foundation radius; $G_{s}, \mu_{s}$ and $\rho_{s}$ are the shear modulus, the Poisson's ratio and the density of the soil body, respectively. 


\subsection{Blade Modelling}

Each rotor blade is simulated to be a rotary cantilever that has a point mass $M_{\text {Tip }}$ fixed at the free end and rotating about an axis perpendicular to the rotor plane at the angular velocity $\Omega$. Suppose the flexible part of each blade moves independently flap-wise (perpendicular to the wing-type string) and span-wise (parallel with the wing-type string). Meanwhile, blade deformation can be expressed as deformation towards the in-plane direction (parallel with the rotor rotating plane) and the out-of-plane direction (perpendicular to the rotor rotating plane). The relationship between the flap-wise-span-wise and in-plane-out-of-plane coordinate systems is shown in Fig. 5.

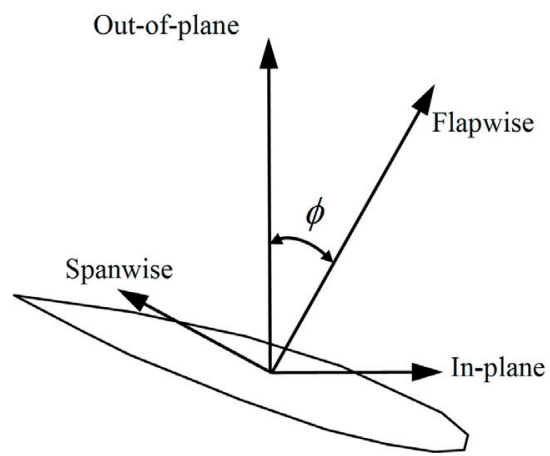

Fig. 5. Relationship between coordinate systems

In the rotor rotation system, the dynamic energy of the blade is consistent with the dynamic energy of the tower. The generalized mass of the blade can be expressed as:

$$
m_{i j}=M_{T i p}+\int_{0}^{R-R_{H}} \mu_{B}(r) \phi_{i}(r) \phi_{j}(r) d r
$$

where $\mu_{B}(\mathrm{r})$ is the blade distribution line density, $R$ is the rotor radius, and $R_{H}$ is the hub radius. When the gravity is neglected, the potential of the blade comprises component $V_{\text {Beam }}$ associating with the blade distribution rigidity and component $V_{\text {Rotation }}$ associating with centrifugal force produced by blade rotation.

$$
V=V_{\text {Beam }}+V_{\text {Rotation }}
$$

The blade potential component associating with the distribution rigidity of the cantilever can be expressed as:

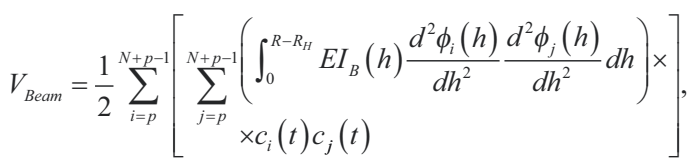

where $E I_{B}(h)$ is the flap-wise or span-wise blade distribution rigidity.

The potential component produced by rotor rotation can be expressed as:

$$
\begin{aligned}
& V_{\text {Rotation }}=\frac{1}{2} \Omega^{2} \times
\end{aligned}
$$

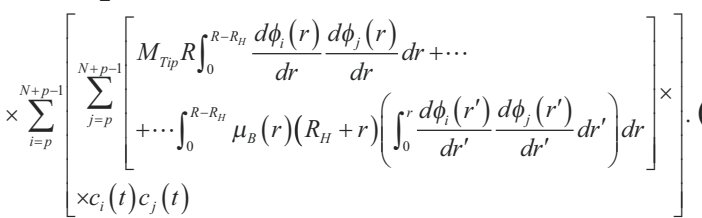

The first item in the bracket is the potential component associating with the mass of the bladetip braking part; the second item is the potential component associating with the blade distribution mass. The positive sum of the centrifugal potential value of the two items is the generalized rigidity to be increased of the blade by the centrifugal force, i.e. centrifugal rigidity.

Through simplification, the generalized rigidity of the blade can be expressed as:

$$
\begin{aligned}
k_{i j}= & \int_{0}^{R-R_{H}} E I_{B}(r) \frac{d^{2} \phi_{i}(r)}{d r^{2}} \frac{d^{2} \phi_{j}(r)}{d r^{2}} d r+ \\
& +\Omega^{2}\left[M_{T i p} R+\int_{r}^{R-R_{H}} \mu_{B}\left(r^{\prime}\right)\left(R_{H}+r^{\prime}\right) d r^{\prime}\right] . \\
& . \frac{d \phi_{i}(r)}{d r} \frac{d \phi_{j}(r)}{d r} d r
\end{aligned}
$$

\section{DYNAMIC MODEL AND SOLUTION}

Suppose the wind turbine is a first order linear multibody system with $N$ freedoms; the motion of the wind turbine system can be described by using $N$ generalized coordinates $q_{i}(i=1,2, \ldots, N)$, or by using $N$ generalized velocities $u_{r}(r=1,2, \ldots, N)$. The latter are $N$ independent scalars selected arbitrarily from the module values of the rigid body angular velocities or the particle velocities comprising the system, and can be expressed as the linear combination of generalized coordinate differential coefficients $\dot{q}_{i}(i=1,2, \ldots, 15)$. For the cabin, it is simplified as a rigid model for calculation.

$$
u_{r}=\sum_{i=1}^{N} Y_{r i} \dot{q}_{i}+Z_{r}, \quad r=1,2, \cdots, N,
$$

where $Y_{r i}$ and $Z_{r}$ are the functions of the generalized coordinate $q_{i}$ and time $t$; if $u_{r}$ is an independent variable, the only solution $\dot{q}_{i}$ to Eq. (20) can be obtained, and we obtain: 


$$
\dot{q}_{i}=\sum_{r=1}^{N} W_{i r} u_{r}+X_{i}, \quad i=1, \cdots, N .
$$

After the generalized velocity is determined, the absolute angular velocity ${ }^{E} \omega^{N_{i}}(\dot{q}, q, t)$ and absolute linear velocity ${ }^{E} v^{N_{i}}(\dot{q}, q, t)$ of the $N_{i}^{\text {th }}$ rigid body in the wind turbine system corresponding to the inertial coordinate system $E$ can be only expressed as a linear combination of $u_{r}$.

$$
\begin{gathered}
{ }^{E} \omega^{N_{i}}(\dot{q}, q, t)=\sum_{r=1}^{N}{ }^{E} \omega_{r}^{N_{i}}(q, t) u_{r}+{ }^{E} \omega_{t}^{N_{i}}(q, t), \\
{ }^{E} v^{N_{i}}(\dot{q}, q, t)=\sum_{r=1}^{N}{ }^{E} v_{r}^{N_{i}}(q, t) u_{r}+{ }^{E} v_{t}^{N_{i}}(q, t),
\end{gathered}
$$

where ${ }^{E} \omega_{r}^{N_{i}}(q, t)$ and ${ }^{E} v_{r}^{N_{i}}(q, t)$ are the $r^{\text {th }}$ angular velocity vector and the $r^{\text {th }}$ linear velocity vector, respectively, of the $N_{i}$ th rigid body in the inertia coordinate system $E$. Based on time derivation, we can obtain the angular acceleration ${ }^{E} \varepsilon^{N_{i}}(\ddot{q}, \dot{q}, q, t)$ and the acceleration ${ }^{E} a^{N_{i}}(\ddot{q}, \dot{q}, q, t)$ of the $N_{i}^{\text {th }}$ rigid body in the inertial coordinate system $E$.

After the partial velocity and angular velocity of each rigid body, as well as the corresponding generalized active force $F$ and the generalized inertia force $F^{*}$ are determined, the Kane kinetic equation can be expressed as:

$$
F_{r}+F_{r}^{*}=0, \quad(r=1,2, \cdots, N),
$$

Suppose the wind turbine system comprises $w$ rigid bodies and for each rigid body $N_{i}$, the active force is applied on the centroid $X_{i}$; the generalized active force of the wind turbine system can be expressed as:

$$
F_{r}=\sum_{i=1}^{w}{ }^{E} v_{r}^{X_{i}} \cdot F^{X_{i}}+{ }^{E} \omega_{r}^{N_{i}} \cdot M^{N_{i}}, \quad(r=1,2, \cdots, 15),
$$

The corresponding generalized inertia force can be expressed as:

$$
\begin{gathered}
F_{r}^{*}=\sum_{i=1}^{w}{ }^{E} v_{r}^{X_{i}} \cdot\left(-m^{N_{i} E} a^{X_{i}}\right)+{ }^{E} \omega_{r}^{N_{i}} \cdot\left(-{ }^{E} \dot{H}^{N_{i}}\right), \\
(r=1,2, \cdots, 15),
\end{gathered}
$$

where

$$
{ }^{E} \dot{H}^{N_{i}}=\left\{\begin{array}{c}
\left(\dot{H}^{N_{i}}\right)^{\prime}+{ }^{E} \omega^{N_{i}} \times{ }^{E} H^{N_{i}} \\
\text { or } \\
\overline{\bar{I}}^{N_{i}} \cdot{ }^{E} \varepsilon^{N_{i}}+{ }^{E} \omega^{N_{i}} \times \overline{\bar{I}}^{N_{i}} \cdot{ }^{E} \omega^{N_{i}}
\end{array} .\right.
$$

Substitute Eq. (25) and Eq. (26) into the Kane kinetic equation (Eq. ((24)), and we can obtain the kinetic equation of the wind turbine system, expressed in matrix as:

$$
[C(q, t)]\{\ddot{q}\}+\{f(\dot{q}, q, t)\}=\{0\},
$$

where $[C(q, t)]$ is the system acceleration coefficient matrix, and $\{f(\dot{q}, q, t)\}$ is the vectors relating to the system displacement and the velocity. To obtain a solution at each time step, the fourth-order AdamsBashforth prediction-correction algorithm is first used at each time step to determine the value of the lower-order item, which constitutes the right item of the equation; then the Gauss elimination method is used to obtain the system freedom acceleration. The acceleration obtained through these calculations is then used to correct the estimated value and promote precision. Through several iterations, the fourth-order Adams-Bashforth prediction-correction algorithm is used to determine the acceleration and the final solution at the time step. As the prediction-correction algorithm is not spontaneous, solutions at the first four time steps shall be determined by using the fourthorder Runge-Kutta method.

\section{EXCITATION EARTHQUAKE LOAD}

In this paper, the seismic load acts on the base of the wind turbine tower system in the form of an acceleration process, while acceleration is generated based on the acceleration response spectrum designed in the structural specifications. In engineering design, Eurocode 8 is widely applied throughout the world. To be universal, this paper obtains the seismic acceleration process based on Eurocode 8, according to which the designed acceleration response spectrum of a seismic load can be expressed [17] as shown in Fig. 6. The calculation formula is shown as follows:

$$
\left\{\begin{array}{c}
S_{e}(T)=a_{g} \cdot S \cdot\left[1+\frac{T}{T_{B}} \cdot(\eta \cdot 25-1)\right], 0 \leq T \leq T_{B} \\
S_{e}(T)=a_{g} \cdot S \cdot \eta \cdot 25, T_{B} \leq T \leq T_{C} \\
S_{e}(T)=a_{g} \cdot S \cdot \eta \cdot 25 \cdot \frac{T_{C}}{T}, T_{C} \leq T \leq T_{D} \\
T_{D} \leq T \leq 40 s, S_{e}(T)=a_{g} \cdot S \cdot \eta \cdot 25 \cdot \frac{T_{C} T_{D}}{T^{2}}
\end{array}\right.
$$

where $\operatorname{Se}(T)$ is the elastic response spectrum; $T$ is the vibration period; $T_{B}$ and $T_{C}$ are the periodical constant range limits of the acceleration spectrum; $T_{D}$ is the start period constant value of the displacement response spectrum; $a_{g}$ is the designed ground 
acceleration of A-type sites; $S$ is the soil-associated coefficient; $\eta$ is the damping correction coefficient, according Eurocode 8, $\quad \eta=\sqrt{10 /(5+\xi)} \geq 0.55$, $\eta=1$ and damping rate $\xi=5$ in this paper. Fig. 7 is the Acceleration process of an earthquake according Fig.

6.

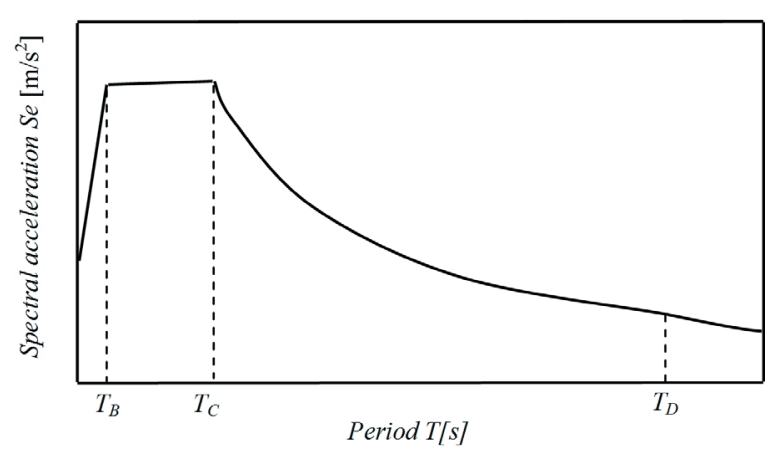

Fig. 6. Earthquake spectrum according Eurocode 8

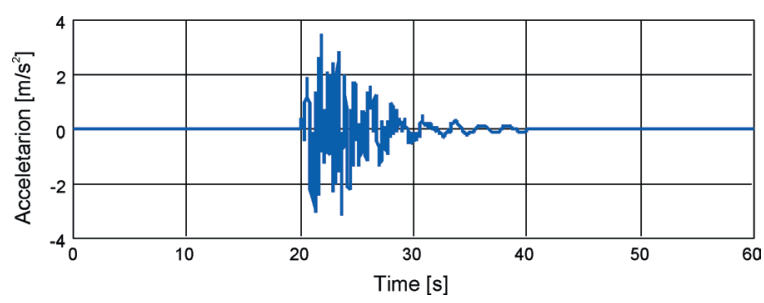

Fig. 7. Acceleration process of an earthquake

\section{EXAMPLE}

\subsection{Seismic Impact on Wind Turbine Performance}

To take into account the seismic impact on a working wind turbine, seismic analyses of the wind turbine are carried out through the establishment of a theoretical model. As most winds in the natural world prevail as irregular turbulent winds, if turbulent winds are adopted in the seismic analyses, the significance of a seismic impact can hardly be identified.

Table 1. Dynamic motion comparison

\begin{tabular}{llccc}
\hline & & $\begin{array}{c}\text { Deformation } \\
{[\mathrm{m}]}\end{array}$ & $\begin{array}{c}\text { Deformation } \\
\text { velocity }\left[\mathrm{m} \cdot \mathrm{s}^{-1}\right]\end{array}$ & $\begin{array}{c}\text { Deformation } \\
\text { acceleration }\left[\mathrm{m} \cdot \mathrm{s}^{-2}\right]\end{array}$ \\
\hline \multirow{2}{*}{$\begin{array}{l}\text { Blade } \\
\text { tip }\end{array}$} & Model & 3.98 & 10.72 & 35.27 \\
\cline { 2 - 5 } $\begin{array}{l}\text { Tower } \\
\text { top }\end{array}$ & Bladed & 4.17 & 11.92 & 37.05 \\
\cline { 2 - 5 } & Model & 0.61 & 1.26 & 2.47 \\
\hline
\end{tabular}

Therefore, to better clarify impact of seismic excitation on the wind turbine, a steady wind at the speed of $11 \mathrm{~m} / \mathrm{s}$ is used in the case described in this paper. To validate the correctness of the model, the calculation results are compared with GH Bladed [18]. It can be seen from the comparison that the calculations using this model are correct, as shown in Table 1.
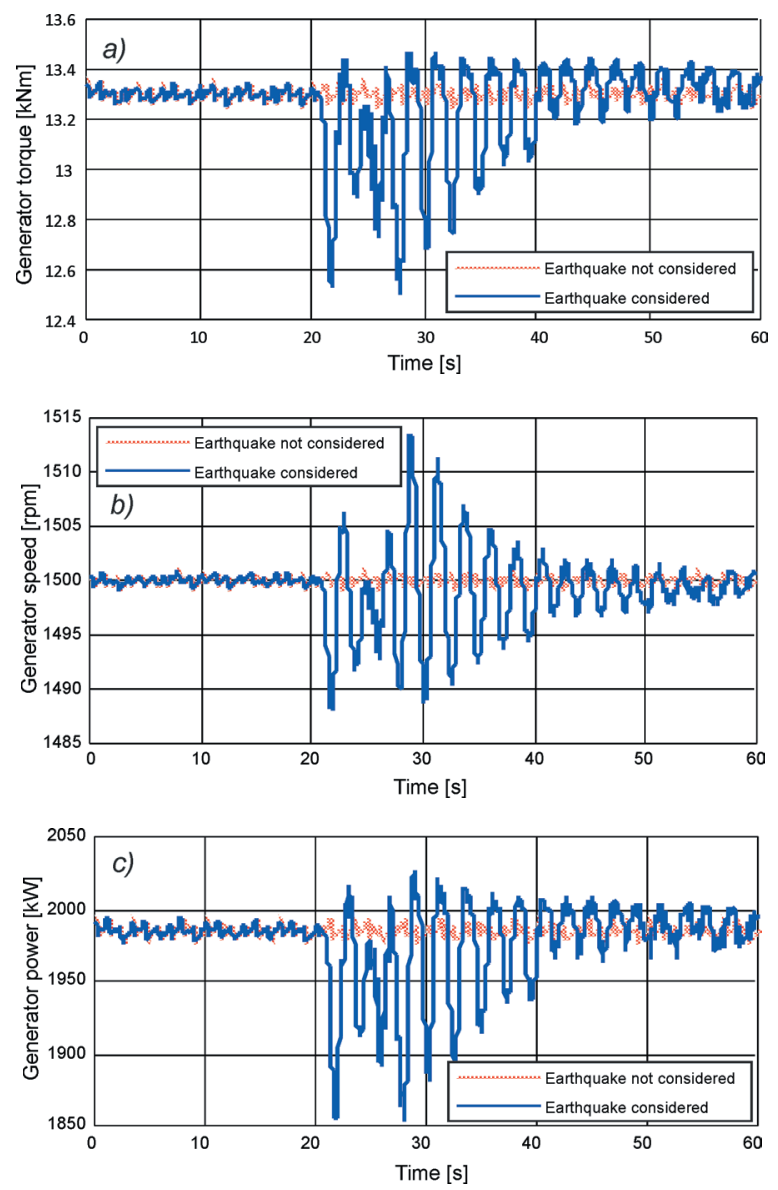

Fig. 8. Seismic impact on the performances of wind turbines; a) generator torque comparison, b) wind turbine speed comparison, and c) wind turbine power comparison

Fig. 8 shows the torque, speed and power of the wind turbine. In the figures, the part in red indicates normal generation without considering seismic impact, and the part in blue indicates normal generation under seismic impact. It can be seen from the three figures that upon application of seismic excitation at the $20^{\text {th }}$ second, the torque, speed and power of the wind turbine are disturbed to different degrees. In Fig. 8a, the disturbance to the wind turbine torque is relatively great, about $6.01 \%$ as calculated, mainly due to minor changes of the incoming wind velocity relative to the blade element upon occurrence of a seismic shock, resulting in a change of the aerodynamic torque; in Fig. 8b, disturbance to the wind turbine speed is relatively small, about $1 \%$ as calculated, mainly due to 
short seismic excitation and inertia of the rotor; in Fig. $8 \mathrm{c}$, disturbance to the wind turbine power is mainly caused by joint action of the wind turbine speed and torque. In general, the seismic shock has no significant impact on wind turbine operation, provided the wind turbine structure is not damaged; whether the structure will be damaged mainly depends on the load caused by the earthquake.

\subsection{Seismic Impact on Load-Bearing Conditions}

Generally, the load on the tower base, spindle and blade root of a wind turbine is the most representative in the load calculation. Therefore, to study the seismic impact on the major load on a wind turbine, three
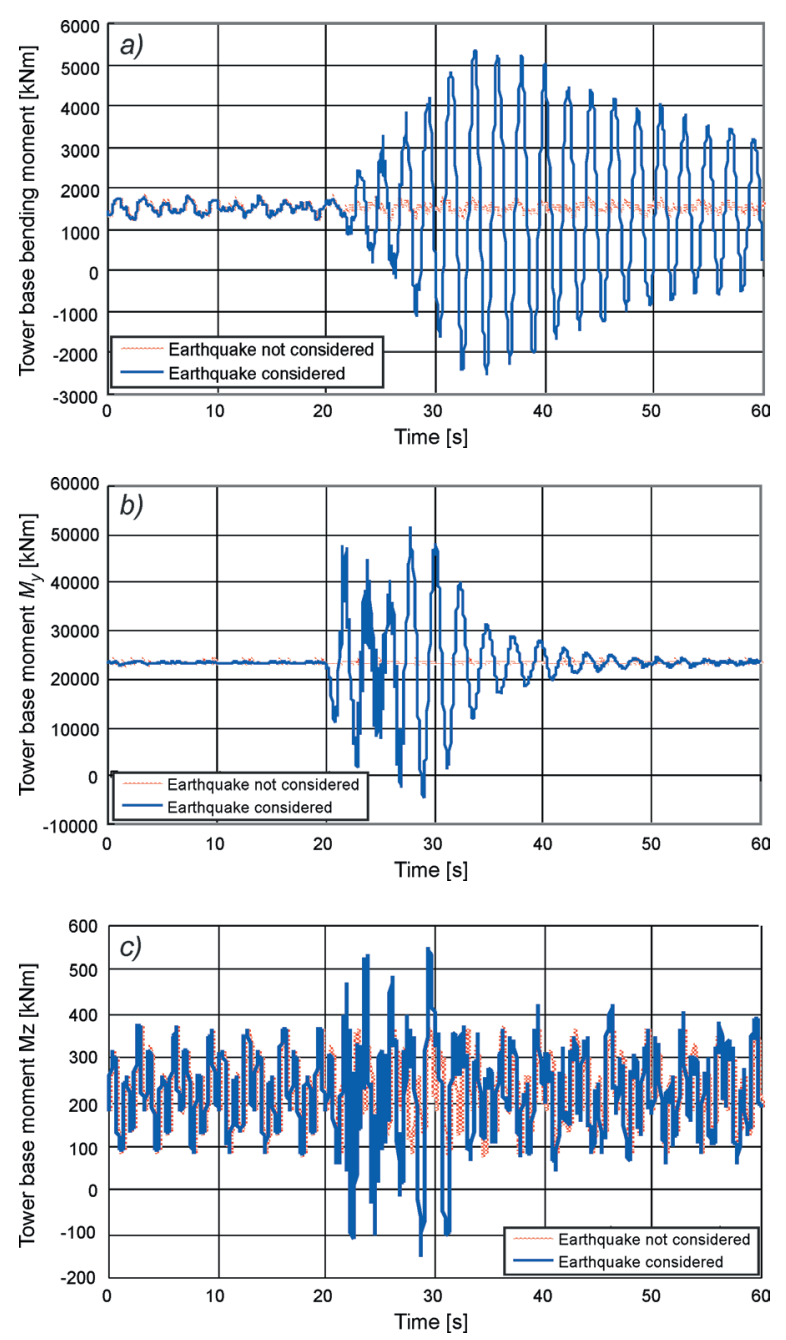

corresponding load groups are described, i.e. the load on the tower base, load on the spindle and load on the blade root (in the seismic propagation order). Similarly, in the figures, the part in red indicates normal generation without considering seismic impact, and the part in blue indicates normal generation under seismic impact. Fig. 9 shows the load on the tower base; Fig. 9a is the tower base bending moment $M_{x}$, with the maximum load fluctuation caused by the seismic shock reaching $188 \%$; Fig. $9 \mathrm{~b}$ is the tower base bending moment $M_{y}$, with the maximum load fluctuation caused by the seismic shock reaching $108 \%$; Fig. $9 \mathrm{c}$ is the tower base bending moment $M_{z}$, with the maximum load fluctuation caused by the seismic shock reaching $45 \%$; Fig. $9 \mathrm{~d}$ is load $F_{x}$ on
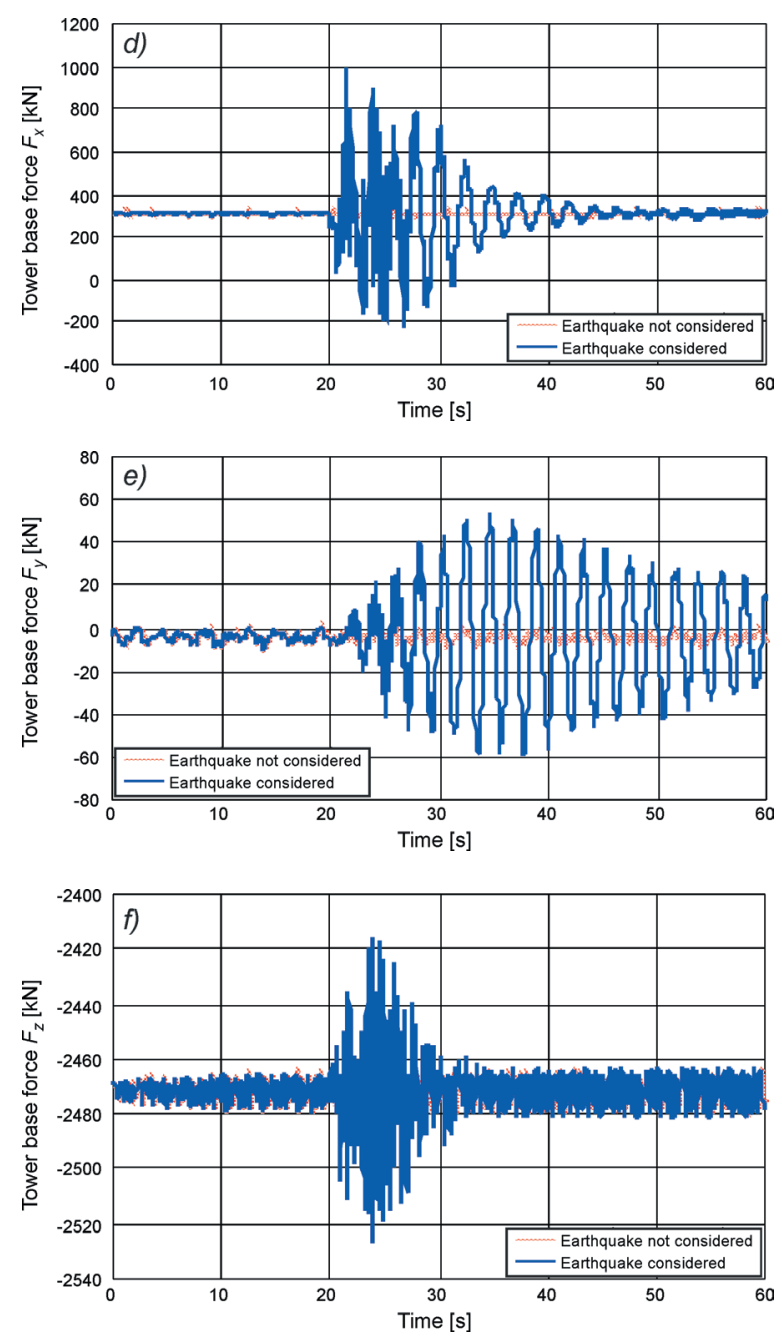

Fig. 9. Seismic impact on load-bearing conditions of the tower base; comparison of: a) foundation bending moment Mx, b) foundation bending moment My, c) foundation bending moment Mz, d) load Fx acting on the base, e) load Fy acting on the base, f) load Fz acting on the base 

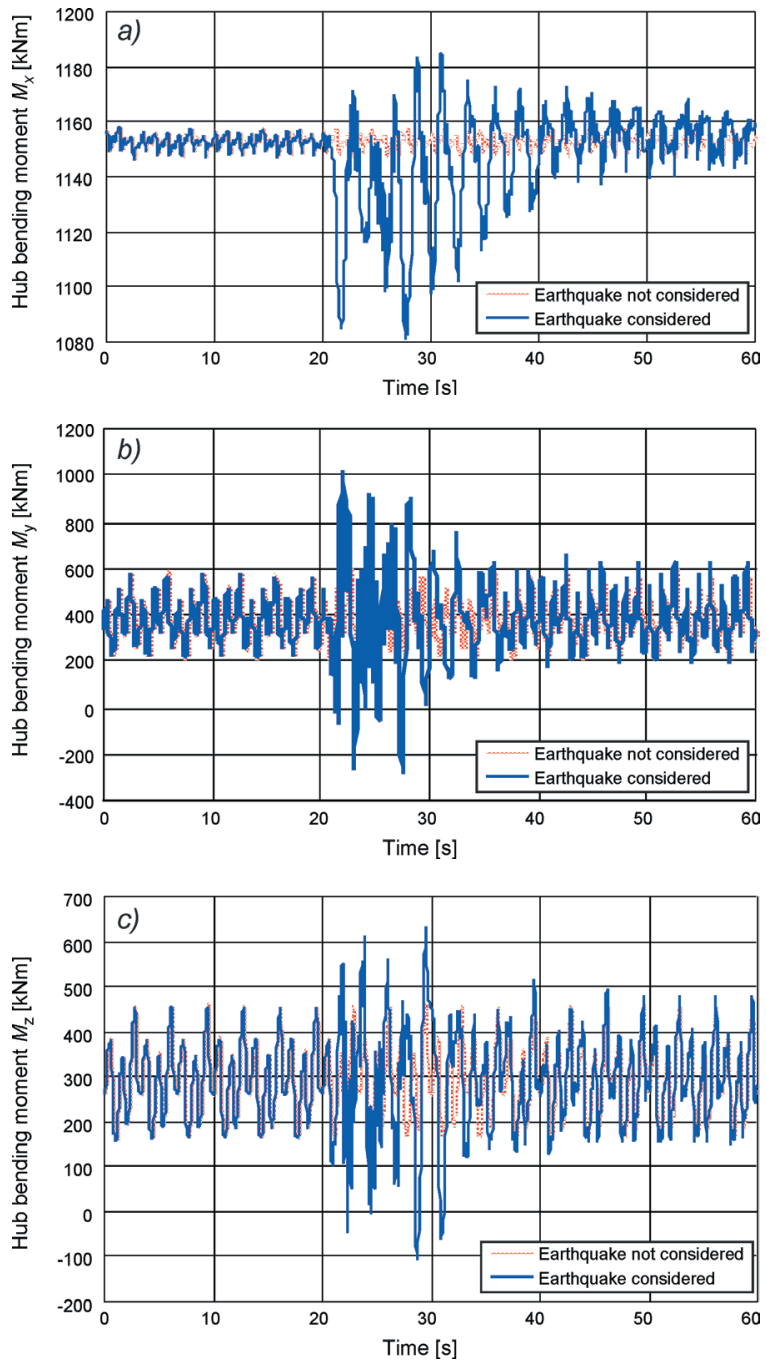
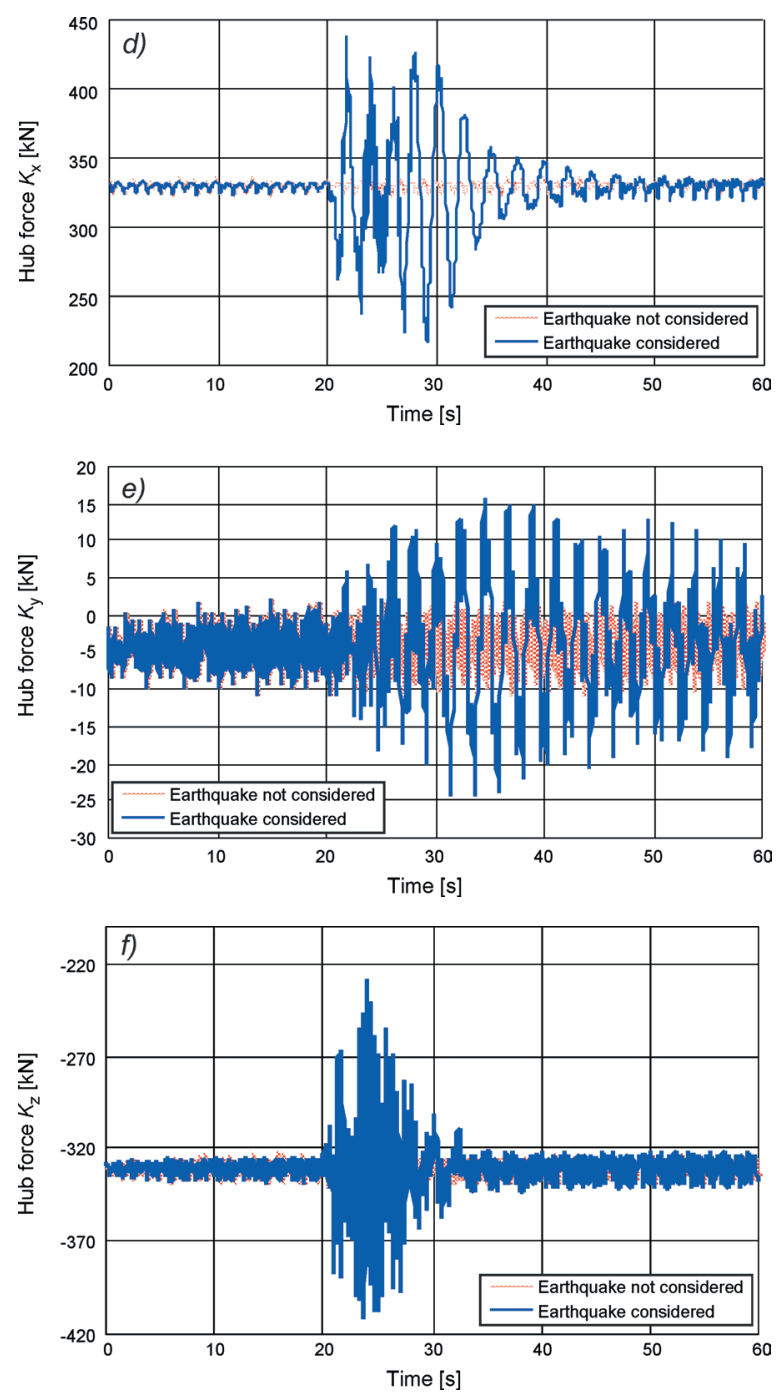

Fig. 10. Seismic impact on load to be borne by the hub; comparison of: a) hub bending moment $M_{x}$, b) hub bending moment $\left.M_{y}, c\right)$ hub bending moment $M_{z}, d$ ) load $F_{x}$ acting on the hub, e) load $F_{y}$ acting on the hub, f) load $F_{z}$ acting on the hub

the tower base, with the maximum load fluctuation caused by the seismic shock reaching 233\%; Fig. $9 \mathrm{e}$ is load $F_{y}$ on the tower base, with the maximum load fluctuation caused by the seismic shock reaching $500 \%$; Fig. 9f is the tower base bending moment $M_{z}$, with the maximum load fluctuation caused by the seismic shock reaching $2.41 \%$. Generally, as the seismic shock first arrives at the tower base, it causes great fluctuation of load on the tower base.

Fig. 10 shows the load on the spindle hub; Fig. 10a is the bending moment $M_{x}$, with the maximum load fluctuation caused by the seismic shock reaching $6.1 \%$; Fig. $10 \mathrm{~b}$ is $M_{y}$, with the maximum load fluctuation caused by the seismic shock reaching $66.7 \%$; Fig. $10 \mathrm{c}$ is the tower base bending moment
$M_{z}$, with the maximum load fluctuation caused by the seismic shock reaching 30\%; Fig. 10d is load $F_{x}$ on the tower base, with the maximum load fluctuation caused by earthquakes reaching 36\%; Fig. 10e is load $F_{y}$ on the tower base, with the maximum load fluctuation caused by the seismic shock reaching $150 \%$; Fig. 10f is the tower base bending moment $M_{z}$, with the maximum load fluctuation caused by the seismic shock reaching $20 \%$. Generally, as the seismic shock first arrives at the tower base, it causes great fluctuation of load on the tower base. The results show that as the tower is a flexible fine long piece, loads caused by seismic excitation will be reduced in the amount and magnitude when arriving at the spindle after passing the tower. 

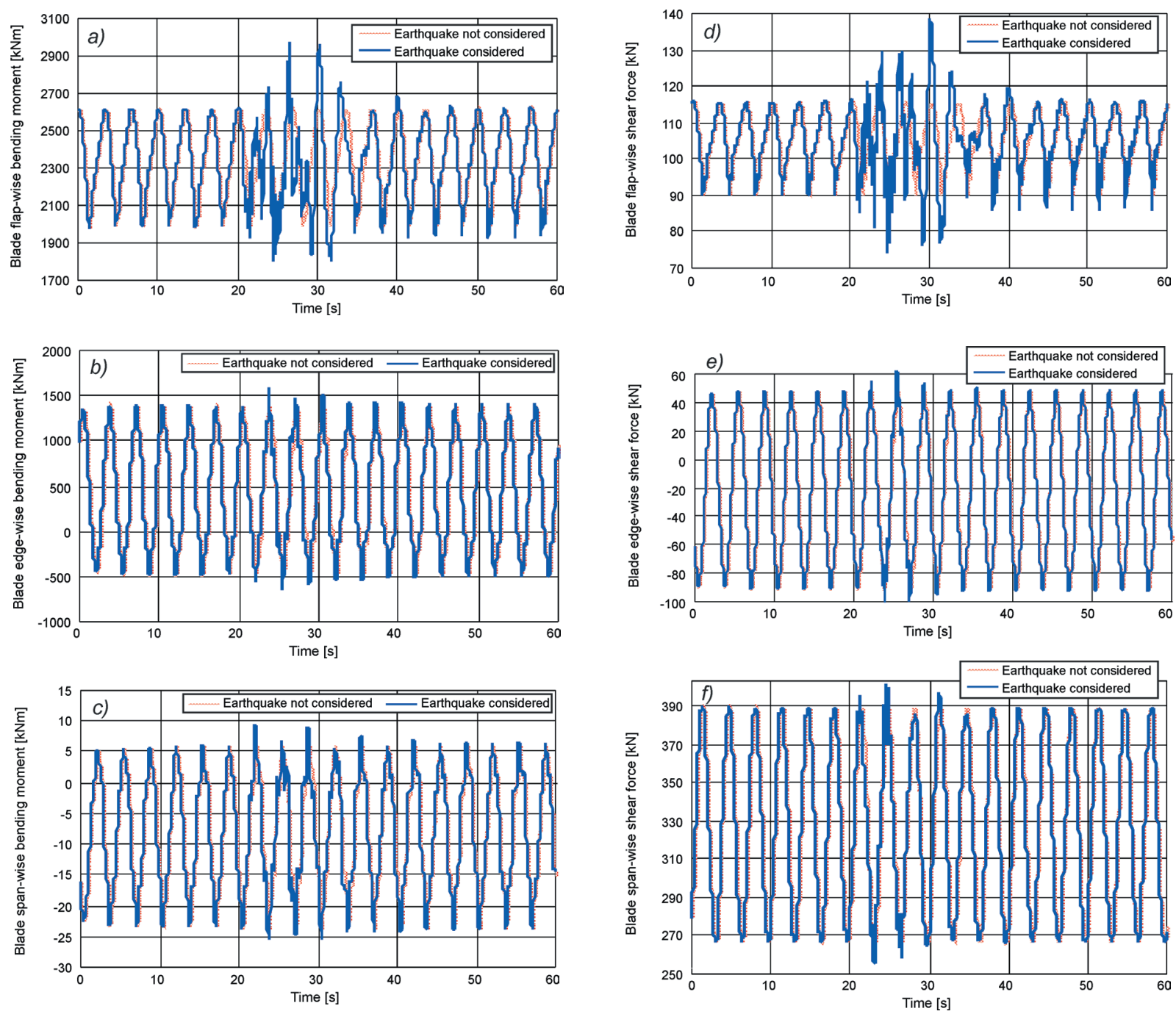

Fig. 11. Seismic impact on blade root load; a) blade flap-wise moment comparison, b) blade edge-wise moment comparison, c) blade spanwise moment comparison, e) blade flap-wise force comparison, d) blade edge-wise force comparison, f) blade span-wise force comparison

Fig. 11 shows the load on the blade root, specifically the flap-wise, edge-wise and span-wise moments and loads, respectively. It can be seen from the figures that, due to seismic impact, load fluctuations only occur in $a$ ) and $e$ ), but the load in other figures is not subject to significant impacts, mainly because the blade is a flexible fine long piece, just as the tower, which reduces the seismic impact.

\section{CONCLUSION}

According to the multi-body dynamic theory this paper proposes a multi-body-system dynamic model based on blade-cabin-tower-foundation coupled multi-body system dynamic model with soil-structure interaction considered to study the load-bearing conditions of wind turbines subject to seismic impact. Based on the basic theory of multi-body system dynamics, the wind turbine blade and tower system comprise a series discrete continuous units, while the soil-structure interaction of the tower system is realized via the spring and damping set on the interface between the foundation and the soil body; the cabin is simplified as a rigid model.

Analyses show that a sudden earthquake occurring during normal operation of a wind turbine will disturb the wind turbine performance to some extent, but the generating performance will not fluctuate greatly, provided that the wind turbine structure is not damaged; if the wind turbine structure is damaged, the disturbance intensity will depend on the load caused by the seismic shock. Through the 
three typical load groups selected for the wind turbine, it can be seen that load on the tower base suffers the greatest fluctuation when it is the nearest to the foundation, which presents higher requirements for the tower base design; the seismic excitation transmits through the tower and then reaches the top of the tower, so the load fluctuation at the spindle reduces greatly; when it reaches the blade, the load fluctuation reduces more significantly as the blade is of a flexible structure. Generally, the seismic shock exerts the most significant impact on the tower base, but much less significant impact on the upper part, which is an advantage of the flexible structure. Therefore, when designing wind turbines for earthquake-prone regions, flexible structures should be considered for vibration relief.

\section{ACKNOWLEDGEMENT}

This paper is sponsored by Natural Science Foundation of China (approval No.: 5100 5255) and the Fundamental Research Funds for the Central Universities. (Approval No.: CDJZR10 1100 04).

\section{REFERENCES}

[1] Stamatopoulos, G.N. (2013). Response of a wind turbine subjected to near-fault excitation and comparison with the Greek Aseismic Code provisions. Soil Dynamics and Earthquake Engineering, vol. 46, p. 77-84, DOI:10.1016/j.soildyn.2012.12.014.

[2] Teng, W., Wang, F., Zhang, K.L., Liu, Y.B., Ding, X. (2014). Pitting fault detection of a wind turbine gearbox using empirical mode decomposition. Strojniški vestnik - Journal of Mechanical Engineering, vol. 60, no. 1, p. 12-20, DOI:10.5545/sv-jme.2013.1295.

[3] Potočar, E., Širok, B., Hočevar, M., Eberlinc, M. (2014). Control of Separation Flow over a Wind Turbine Blade with Plasma Actuators. Strojniški vestnik - Journal of Mechanical Engineering, vol. 58, no.1, p. 37-45, DOI:10.5545/sv-jme.2011.016.

[4] DNV/Risø National Laboratory. (2001). Guidelines for design of wind turbines. 2nd ed., Wind Energy Department, Roskilde.

[5] Bazeos, N., Hatzigeorgiou, G.D., Hondros, I.D., Karamaneas, H., Karabalis, D.L., Beskos, D.E. (2002). Static, seismic and stability analyses of a prototype wind turbine steel tower. Engineering Structures, vol. 24, no. 8, p. 1015-1025, DOI:10.1016/S01410296(02)00021-4.
[6] Lavassas, I., Nikolaidis, G., Zervas P., Efthimiou, E., Doudoumis, I.N., Baniotopoulos, C.C. (2003). Analysis and design of the prototype of a steel 1-MW wind turbine tower. Engineering Structures, vol. 25, no. 8, p. 1097-1106, DOI:10.1016/S0141-0296(03)00059-2.

[7] Murtagh, P.J., Basu, B., Broderick, B.M. (2005). Along-wind response of a wind turbine tower with blade coupling subjected to rotationally sampled wind loading. Engineering Structures, vol. 27, no. 8, p. 12091219, DOI:10.1016/j.engstruct.2005.03.004.

[8] Murtagh. P.J., Basu, B., Broderick, B.M. (2005). Along-wind response of a wind turbine tower with blade coupling subjected to rotationally sampled wind loading. Engineering Structures, vol. 27, no. 8, p. 12091219, DOI:10.1016/j.engstruct.2005.03.004.

[9] Witcher, D. (2005). Seismic analysis of wind turbines in the time domain. Wind Energy, vol. 8, no. 1, p.81-91, DOI:10.1002/we.135.

[10] Kang, H., Li, Y., Wu, F., Guo, W., Huan, K. (2008). A system reliability analysis method for offshore wind turbine foundation. Electronic Journal of Geotechnical Engineering, vol. 13, bundle L, p. 1-10.

[11] Harte, M., Basu, B., Nielsen, S.R.K. (2012). Dynamic analysis of wind turbines including soil-structure interaction. Engineering Structures, vol. 45, p. 509518, DOI:10.1016/j.engstruct.2012.06.041.

[12] Lombardi, D., Bhattacharya, S., Wood, D.M. (2013). Dynamic soil-structure interaction of monopile supported wind turbines in cohesive soil. Soil Dynamics and Earthquake Engineering, vol. 49 p. 165-180, DOI:10.1016/j.soildyn.2013.01.015.

[13] Newmark, N.M., Rosenblueth, E. (1971). Fundamentals of Earthquake Engineering. Prentice Hall, New Jersey.

[14] Stejska, J.V., Valasek, M. (1996). Kinematics and dynamics of machinery. Marcel Dekker, Inc., New York.

[15] Wolf, J.P. (1997). Spring-dashpot-mass models for foundation vibrations. Journal of Earthquake Engineering \& Structural Dynamics, vol. 26, no. 9 , p. 931-949, DOI:10.1002/(SICI)10969845(199709)26:9<931::AID-EQE686>3.0.CO;2-M

[16] Mulliken, J.S., Karabalis, D.L. (1998). Discrete model for dynamic through-the-soil coupling of 3-D foundations and structures. Earthquake Engineering \& Structural Dynamics, vol. 27, no. 7 , p. 687-710, DOI:10.1002/(SICI)10969845(199807)27:7<687::AID-EQE752>3.0.CO;2-O.

[17] European Committee for Standardization (2003). Eurocode8: Design of Structure for Earthquake Resistance. London South Bank University, London,

[18] Bladed, Wind Turbine Design Software (2014). from http://www.gl-garradhassan.com/en/softwarel GHBladed.php, accessed on 2014-01-04. 